\title{
Pemphigus vulgaris, myasthenia gravis and membranous colitis
}

\author{
C. M. RIDLEY \\ B.M., M.R.C.P. \\ Consultant Dermatologist, Whittington Hospital, London, N.19
}

\section{Introduction}

A leading article in the Lancet (1968) discussed evidence tending to suggest a significant association between myasthenia gravis and pemphigus.

The case is reported of a white non-Jewish woman who died at the age of $\mathbf{4 4}$ with pemphigus vulgaris, myasthenia gravis and membranous colitis.

\section{Case report}

In May 1965 the patient, then aged 38, was referred to Dr E. R. Bickerstaff, Consultant Neurologist, because for 3 years she had had weakness of the right hand and arm, worse in the evening, brought on by exercise, and improving with rest although even after rest she tended to drop things easily; her legs felt weak on walking; and she had had some double vision, especially following previous pregnancies, and recent difficulty in speaking and swallowing. She was found to have some weakness of the facial muscles, back muscles, medial fingers of both hands and proximal leg muscles. She was pregnant so further investigations were not considered justifiable. A clinical diagnosis of myasthenia gravis was made and substantiated by a good response to prostigmine $15 \mathrm{mg}$ q.i.d. and later to pyridostigmine $60 \mathrm{mg}$ q.i.d.

Early in 1967 she developed itching blisters on the trunk, especially affecting the waist, groins and axillae; lesions also developed in the vulval area, mouth, eyes and nose. In July 1967, an initial diagnosis of Behçet's disease having been made, systemic steroids were given with good effect. In December 1967, she noticed the growth of hair on the face and pain in the back. She seemed to have become dependent upon methaqualone (Mandrax) and was depressed-in addition to the effects of the physical illness and its treatment there was considerable domestic stress. An X-ray revealed a fractured rib, and some osteoporosis of the spine without collapse. In July 1968 she was transferred at the wish of her own doctor and the consultants concerned to Manor House Hospital, and thence to the Whittington Hospital under the care of $\mathrm{Dr}$ Michael Ashby.

On examination. She was having steroid and antibiotic preparations locally, frusemide, methaqualone, pyridostigmine $60 \mathrm{mg}$ q.i.d., ACTH 15 units i.m. twice daily and betamethasone $0.75 \mathrm{mg}$ daily. She was ill, depressed and grossly Cushingoid in appearance with facial acne and hirsuties and generalized hyperpigmentation. The blood-pressure was $130 / 80$ $\mathrm{mmHg}$, the jugular venous pressure raised, and there was mild oedema of the legs. The liver edge could be felt. There was weakness and wasting of the proximal muscles of the upper limbs, rather more marked on the right; and similar weakness and wasting proximally in the lower limbs, especially on the left. The reflexes were equal, touch and sensation normal and the plantar responses equivocal. There was tenderness over the fourth and fifth lumbar vertebrae. On the skin were widespread raw areas without scarring or milia; Nikolsky's sign was positive. The mouth was similarly affected and there were vegetative lesions of the genito-crural folds. Many nails were missing or grossly dystrophic. There were no lesions clinically suggestive of cutaneous lupus erythematosus or of pemphigus erythematosus (Senear-Usher syndrome).

Investigation. Haemoglobin $93 \%$, WBC 14,400/ $\mathrm{mm}^{3}$ (polymorphs 69\%) and ESR (Westergren) $12 \mathrm{~mm} / \mathrm{hr}$; blood group $\mathrm{O}$, Rhesus positive; serum potassium $2.8 \mathrm{mEq} / \mathrm{l}$ (rising after oral potassium to 3.5 and $4.5 \mathrm{mEq} / \mathrm{l}$ ); the plasma proteins were $5.7 \mathrm{~g} / 100 \mathrm{ml}$-albumin $3.2 \mathrm{~g}$, globulin $2.5 \mathrm{~g}$-with a normal electrophoretic strip. The urine was normal. A chest X-ray showed no abnormality apart from an old fracture of the fifth left rib. X-ray of the spine showed loss of bone density, with compression of the bodies of the second, fourth and fifth lumbar vertebrae. The histology of an excised bulla on the thigh showed an intra-epidermal suprabasal bulla with acantholysis, confirming the clinical diagnosis of pemphigus vulgaris.

Progress. Immediately after admission, the betamethasone was slowly reduced, though not the ACTH, because of the apparently poor response to treatment and the side-effects of the therapy.

During this period she developed a high temperature and rigors. Blood cultures grew haemolytic streptococci, Lancefield Group A. She responded to treatment with cloxacillin, ampicillin and crystalline penicillin. Two subsequent blood cultures were negative. 
The skin lesions became worse, and once the diagnosis of pemphigus vulgaris had been established by biopsy large doses of prednisone were given. Control of the lesions was achieved with ACTH 100 units i.m. prednisone $200 \mathrm{mg}$ daily. Alkalies, methandienone (Dianabol), calcium gluconate (Calcium Sandoz) and potassium chloride (Slow K) were given as well. Pellets of betamethasone 17-valerate were used in the mouth, and local nystatin for a monilial infection there.

At this stage the patient developed, over a period of a few days, lower abdominal pain, spreading to the right flank and associated with distension of the abdomen and the frequent passage of fresh blood per rectum. There was a little nausea and no vomiting. Proctoscopy was normal except for the presence of anchovy-like stool, and sigmoidoscopy showed a granular appearance of the rectal wall and some blood in the rectum. The X-rays of the abdomen showed fluid levels in the large and small bowel. The distension and bleeding persisted, shifting dullness developed and the bowel sounds became obstructive. The blood-pressure over this period was $160 / 100$ to $170 / 110 \mathrm{mmHg}$ - higher than the previous levels. The white blood cells were $45,000 /$ $\mathrm{mm}^{3}$ (polymorphs $90 \%$ ) and the platelets normal. At first a provisional diagnosis of a steroid-induced peptic ulcer had been made, but the possibility of a small bowel infarction was later considered more likely. In spite of the hazards of anaesthesia and operation in such a case a laparotomy appeared to be unavoidable.

Operation. With increased steroids (prednisone $60 \mathrm{mg}$ q.i.d. i.m., ACTH 100 units daily i.m., and four doses of hydrocortisone, $100,50,50$ and $25 \mathrm{mg}$ 12 hourly i.v.) increased therapy for the myasthenia gravis (neostigmine $1 \mathrm{mg}$ q.i.d. i.m. and atropine $0.2 \mathrm{mg}$ q.i.d. i.m.), and systemic ampicillin, the operation and immediate post-operative period were uneventful. At laparotomy (Mr J. M. Davis) all the colic vessels were pulsating normally. The colon was oedematous, with intramural and subserous haemorrhages, from the caecum to $3 \mathrm{in}$. below the splenic flexure; the changes were maximal in the caecum. There was about a pint of free peritoneal fluid. The fluid proved sterile on culture. Four inches of the terminal ileum, and the colon to the midsigmoid area, were excised and the ileum joined to the mid-sigmoid colon by an end-to-end anastomosis. It was considered preferable to carry out this procedure, rather than an ileostomy and colostomy, because of the potentially bullous state of her skin.

The excised bowel showed normal terminal ileum and ileo-caecal valve, and a thin-walled friable colon. The colonic mucosal surface was dark red and covered with patches of cream-coloured slough, readily peeling off. The changes faded away at the upper limit of the sigmoid.

Histology (Dr Basil Morson). The ileum was normal but the colon showed extensive recent intramural haemorrhage, mostly in the submucosal layer but with a little in the muscularis propria. There were degenerative changes in the muscle, and patch fullthickness loss of the mucosa. Areas of mucosa which remained showed recent fibrin thrombus in the capillaries, and fibrin mixed with pus cells streaming out to form a membrane on the surface, as well as atrophy, vascular congestion and intramucosal haemorrhage. The sigmoid showed only occasional tiny areas of membrane formation, but vascular congestion and atrophy in the rest of its mucosa. The pathology was thought characteristic of membranous colitis, but the vascular congestion, intramural haemorrhage and degenerative changes in the muscularis were unusual features.

Six days after operation the pemphigus remained suppressed, and the steroids had been slowly reduced to ACTH 100 units daily and prednisone $190 \mathrm{mg}$ i.m. daily. The blood-pressure was $220 /$ $110 \mathrm{~mm} / \mathrm{Hg}$. She then developed right shoulder pain, and pain and distension in the abdomen; X-ray showed consolidation of the right lower lobe, free gas under the diaphragm and free fluid in the peritoneal cavity. Her pulse rate rose and the bloodpressure fell; and, again with further steroid supplements, a second laparotomy was performed. It was found that the anastomosis had broken down and there was diffuse peritonitis. An ileostomy was made on the right side of the abdomen and the sigmoid colon was brought out on the left abdomen but she did not recover and died the next day.

Necropsy (Dr A. J. M. Reese) showed, in addition to the signs of peritonitis, some blood-stained effusion in the pleural cavities, some clear pericardial fluid, hypertrophy of both right and left ventricles, and collapse at the base of the right and left lungs. There was a little fat necrosis in the pancreas. The brain was oedematous, but the meninges, venous sinuses, cerebral arteries and middle ears were normal. The remains of the intestinal tract were normal, as were the kidneys, ovaries, and pituitary gland. The thyroid and suprarenals were enlarged (the latter to twice normal size); fatty tissue only and no tumour was seen at the site of the thymus.

Histology showed autolysed liver, normal thyroid, adipose tissue at the site of the thymus, normal ovary, and hypertrophy of the left ventricular myocardium.

\section{Discussion 1 ,}

This patient had three relatively rare diseases. The question arises as to whether or not the association between any or all of them was other than purely fortuitous. 
Membranous colitis (often referred to as pseudomembranous colitis) is a recently described condition regarded by gastro-enterologists and pathologists as an entity, albeit one of as yet unknown aetiology.

Goulston \& McGovern (1965) survey the literature on the subject and report on fourteen cases. Although admitting that 'mystery and controversy still surround the entity of pseudo-membranous enterocolitis' they give a clear account of the characteristic pathological changes which in their view justify its separate recognition.

The fourteen cases of these authors could be separated into five patients in whom there was preceding colonic disease, where the precipitating factor seemed to be obstruction, and nine patients where there was preceding serious disease in other systems. All presented with diarrhoea, some with pain and some with rectal bleeding. None is noted to have had steroids.

Our patient had an episode of septicaemia, but it seemed to resolve completely some 3 weeks before the bowel symptoms began. No sign was found of any preceding bowel disease on examination of the removed specimen, or at necropsy. The findings were compatible with a diagnosis of membranous colitis, although there were unusual features suggesting a vascular basis for the pathology. It seems likely that the effect of pemphigus vulgaris and perhaps also that of high doses of steroids was responsible for the colitis. The account by Lever (1965) of the causes of death in his own patients with all types of pemphigus before and after the introduction of steroid therapy, and of the sideeffects of steroid therapy when used, does not include anything similar.

There remains to be considered the significance, if any, of the association between myasthenia gravis (MG) and pemphigus vulgaris (PV).

There is good evidence that both have features which in the present state of knowledge lead to their classification as autoimmune diseases. It is thought that diseases in which autoimmunity is a feature tend to be associated together more often than one can ascribe to chance.

Glynn \& Holborrow (1965) and Osserman \& Strauss (1965) describe the evolution of views on the nature of MG and the evidence for its being an autoimmune disease.

Antibodies to the intercellular substance of stratified squamous epithelium in the sera of patients with PV were found by Beutner \& Jordan (1964) using the technique of indirect immunofluorescence.

Since the histological finding regarded as diagnostic of pemphigus vulgaris is acantholysis of epidermal cells leading to the formation of a bulla (Lever, $1965)$ the antigen-antibody reaction may well be of central significance in the disease process, whether it be a consequence or a cause. Certainly the antibodies seem to be closely linked with the clinical findings (Chorzelski, Von Weiss \& Lever, 1966). The literature which has grown up on the subject is summarized and reviewed by Sneddon (1968), Lancet (1968) and Ablin (1968).

Although it seems that PV and MG have not previously been described together, glimpses of a significant relationship appear when one considers PV in connection with pemphigus erythematosus (PE) and PE in connection with MG.

Pemphigus erythematosus, or the Senear-Usher syndrome, is a disease characterized, as is PV, by acantholysis; but the acantholysis in PE occurs in the subcorneal part of the epidermis whereas in PV it occurs in the suprabasal part.

Chorzelski, Jablónska \& Blaszczyk (1968) describe six cases of PE; all six patients showed immunological findings in the epidermis suggestive of PV and five out of six findings suggestive of lupus erythematosus (LE); one of these had coexistent clinical SLE. Investigations into cases of LE and control patients substantiated the validity of these findings. Six cases of PV showed no immunological findings suggestive of LE. One patient had a malignant thymoma, MG and PE and has been described in further detail by Beutner et al. (1968).

In their survey of the literature, the authors note the existence of another (Polish) case with MG and pemphigus (type unspecified) and mention a study in New York by Osserman \& Peck (unpublished) which has revealed three similar cases.

A significant association is suspected to exist between MG and LE. Cases where the two conditions have been combined have been reported by Funkhouser (1961), Alarcon-Segovia et al. (1963), Goldin \& Robbins (1963), Larssen (1963) and Ziff (1963). Mäkelä et al. (1964) review the literature and add a case.

The findings of Chorzelski et al. (1968) and Beutner et al. (1968) go far to link significantly MG and PE, and to show that PE may represent, in immunological terms at least, PV in some way combined with LE. Such a combination of PV and LE in making up PE was suggested by Senear \& Usher (1926) in their original description of PE, in a paper indeed entitled 'An unusual type of pemphigus combining features of LE'. Other authors describe examples of the condition which clinically suggested the combination of LE and PV. Subsequently, however, with the acceptance of acantholysis as the hall-mark of all forms of pemphigus, a process traced by Lever (1965), and the emphasis on histological distinctions in separating disease entities in the bullous dermatoses, it seemed as if PE, where acantholysis occurs higher up in the epidermis than 
in PV, should be classified as a disease separate from PV. PE looks different clinically and has a benign course, and no predilection, as has PV, for the Jewish race. Transitions between PV and PE seem not to occur. Now the wheel has come almost full circle.

One may only speculate on the integration of such knowledge with current views on the status of other forms of acantholytic disease-pemphigus vegetans, histologically and clinically likely to be a variant of PV, pemphigus foliaceus similarly apparently related to $P E$, and Brazilian pemphigus where epidemiology suggests an infective cause. Meanwhile, one may note that Beutner et al. (1965) found similar antibodies to the intercellular substance of the epidermis in sixteen cases of PV, two of pemphigus vegetans, one of $P E$ and one of pemphigus foliaceus; and conclude as do Beutner et al. (1968) that these antibodies may be produced by somewhat different processes in the different forms of pemphigus. Antibodies, moreover, may not be the only result of such processes. The phenomena of autoimmunity may provide stimuli for further work and hypotheses, and its terminology may be a useful language, but only, it seems, by way of such qualifications and reservations will the indisputable clinical findings be accounted for.

The patient here reported had myasthenia gravis and pemphigus vulgaris. Immunological studies unfortunately were not carried out, but the clinical and pathological findings are reported since the association may prove, when further knowledge has been accumulated, to be a significant one.

\section{Acknowledgments}

I should like to thank all those colleagues, senior and junior, who helped in the care of the patient; in particular, Dr Michael Ashby, under whose care she was admitted to the Whittington Hospital, and Mr J. M. Davis, both of whom kindly read the manuscript. I am also grateful to Dr R. G. Ball, the patient's family doctor, and to Dr E. R. Bickerstaff, consultant neurologist, for supplying information about the patient's previous history; and to Miss Jennifer Dennis and Miss Rosalind Newton for secretarial assistance.

\section{References}

ABLIN, R.J. (1968) Autoallergy in pemphigus (Correspondence). Lancet, ii, 1397.

Alarcon-Segovia, D., Galbraith, R.F., Maldonado, J.E. \& Howard, F.M. (1963) S.L.E. following thymectomy for myasthenia gravis. Lancet, ii, 662 .

BeutNer, E.H. \& JoRdon, R.E. (1964) Demonstration of skin antibodies in sera of pemphigus vulgaris patients by indirect immunofluorescent staining. Proceedings of the Society for Experimental Biology and Medicine, 117, 505.

BeutNer, E.H., LeVer, W.F., WitebsKy, E., JoRdon, R. \& Chertock, B. (1965) Autoantibodies in pemphigus vulgaris. Journal of the American Medical Association, 192, 682.

Beutner, E.H., Chorzelski, T.P., Hale, W.L. \& Hausmanowa-Petrusewicz, I. (1968) Auto-immunity in concurrent myasthenia gravis and pemphigus erythematosus. Journal of the American Medical Association, 203, 845.

Chorzelski, T.P., VoN Weiss, J.F. \& LeVer, W.F. (1966) Clinical significance of autoantibodies in pemphigus. Archives of Dermatology, 93, 570.

Chorzelski, T., JablonsKa, S. \& BlaszczyK, M. (1968) Immunopathological investigations in the Senear-Usher syndrome (co-existence of pemphigus and lupus erythematosus). British Journal of Dermatology, 80, 211.

FunkHouser, J.W. (1961) Thymoma associated with myocarditis and the L.E. phenomenon. New England Journal of Medicine, 264, 34.

GlynN, L.E. \& Holborrow, E.J. (1965) Autoimmunity and Disease, Chapter 5. Blackwell Scientific Publications, Oxford and Edinburgh.

Goldin, H. \& RobBINs, W.C. (1963) A patient with myasthenia gravis, thymoma, and lupus nephritis. Arthritis and Rheumatism, 6, 272.

Goulston, S.J.M. \& McGovern, V.J. (1965) Pseudomembranous colitis. Gut, 6, 207.

Lancet (1968) Autoallergy in pemphigus. ii, 1175.

LARSSEN, O. (1963) Thymoma and systemic lupus erythematosus in the same patient. Lancet, ii, 665 .

Lever, W.F. (1965) Pemphigus and Pemphigoid. Thomas, Springfield, Illinois.

Makela, T.E., Ruosteenoja, R., Wager, O., Wallgren, G.R. \& JokINEN, E.J. (1964) Myasthenia gravis and systemic lupus erythematosus. Acta Medica Scandanavica, 75, 777.

Osserman, K.E. \& Strauss, A.J.L. (1965) Immunological Diseases (Ed. by M. Samter and H.L. Alexander), p. 913. Churchill, London.

Senear, F.E. \& Usher, B. (1926) An unusual type of pemphigus combining features of lupus erythematosus. Archives of Dermatology and Syphilis, 13, 761.

SNEDDON, I.B. (1968) Immunological aspects of pemphigus and pemphigoid. British Journal of Dermatology, 80, 410.

ZIFF, M. (1963) in discussion. Arthritis and Rheumatism, 6, 526. 